\title{
Correction to: Solving an Infinite-Horizon Discounted Markov Decision Process by DC Programming and DCA
}

Vinh Thanh Ho and Hoai An Le Thi

\section{Correction to:}

Chapter "Solving an Infinite-Horizon Discounted Markov

Decision Process by DC Programming and DCA" in:

T.B. Nguyen et al. (eds.), Advanced Computational

Methods for Knowledge Engineering, Advances in Intelligent Systems and Computing 453, https://doi.org/10.1007/978-3-319-38884-7_4

In the original version of this chapter, a reference to an earlier chapter was omitted. The reference "Piot, B., Geist, M., Pietquin, O.: Difference of convex functions programming for reinforcement learning. In: Ghahramani, Z., Welling, M., Cortes, C., Lawrence, N.D., Weinberger, K.Q. (eds.) Advances in Neural Information Processing Systems 27, pp. 2519-2527. Curran Associates, Inc. (2014)" has now been added. 